\begin{tabular}{|c|c|}
\hline Title & Unique diffusion behavior observed in supercritical ethanol \\
\hline Author(s) & Ghosh, Swapan K.; T sujii, Kaoru \\
\hline Citation & $\begin{array}{l}\text { Journal of Chemical Physics, 132(14), } 144503 \\
\text { https://doi.org/10.1063/1.3373404 }\end{array}$ \\
\hline Issue Date & $2010-04-14$ \\
\hline Doc URL & http:/hdl .handle.net/2115/43029 \\
\hline Rights & $\begin{array}{l}\text { Copyright } 2010 \text { A merican Institute of Phy sics. This article may be downloaded for personal use only. A ny other use } \\
\text { requires prior permission of the author and the A merican Institute of Phy sics. The following article appeared in J. } \\
\text { Chem. Phys. 132, 144503 (2010) and may be found at https:/dx.doi.org/10.1063/1.3373404 }\end{array}$ \\
\hline Type & article \\
\hline File Information & JCP132-14_144503.pdf \\
\hline
\end{tabular}

Instructions for use 


\title{
Unique diffusion behavior observed in supercritical ethanol
}

\author{
Swapan K. Ghosh ${ }^{1,2, a)}$ and Kaoru Tsujii ${ }^{3}$ \\ ${ }^{1}$ Supramolecular Science Laboratory, RIKEN (The Institute of Physical and Chemical Research), \\ 2-1 Hirosawa, Wako-shi, Saitama 351-0198, Japan \\ ${ }^{2}$ Extremobiosphere Research Center, Japan Agency for Marine-Earth Science and Technology (JAMSTEC), \\ 2-15 Natsushima-cho, Yokosuka-shi, Kanagawa 237-0061, Japan \\ ${ }^{3}$ Research Institute for Electronic Science, Hokkaido University, N21 W10 Kita-ku, Sapporo 001-0021, \\ Japan
}

(Received 18 September 2009; accepted 5 March 2010; published online 9 April 2010)

\begin{abstract}
We have systematically investigated the diffusion behavior of silica nanoparticles within supercritical ethanol, in terms of solvent properties by varying temperature $(T)$ and pressure $(P)$, to elucidate how the inhomogeneous solvent structures and density fluctuations in the solvent affect the diffusion behavior of solute particles. Results show that at a constant pressure, the diffusion coefficient $(D)$ of the particles increases with increasing temperature, reaches the maximum $\left(D^{\max }\right)$ within the gaslike supercritical fluid (slightly below the ridge), and finally decreases abruptly at very low fluid density when temperature is increased further. Results reveal that $D$ is appreciably larger than the theoretical prediction (Einstein-Stokes relationship) in the vicinity of the critical density $\left(\rho_{c}\right)$ of the solvent. We interestingly observed that $D$ becomes maximum $\left(D^{\max }\right)$ at a particular thermodynamic condition $\left(T_{i}, P_{i}\right)$, which is expressed by the empirical formula $T_{r i}=P_{r i}^{0.16}$ (for $T_{r i}>1, P_{r i}>1$ ). Here, $T_{r i}=T_{i} / T_{c}$ and $P_{r i}=P_{i} / P_{c} ; T_{c}$ and $P_{c}$ are the temperature and the pressure at critical point, respectively. Results further reveal that $D^{\max }$ increases significantly with decreasing solvent density within the gaslike supercritical fluid where the changes in viscosities are negligible. These findings are unique, novel, and intriguing. We suggest that the enhancement of the diffusion coefficient in the vicinity of the critical density and the abrupt decrease in the diffusion coefficient in very low density gaslike fluid are associated with the change in the solvent-solvent and solute-solvent direct correlation function (related to the effective interaction potential) upon density change when the fluid crosses the ridge of density fluctuations and within the gaslike fluid.
\end{abstract}

(C) 2010 American Institute of Physics. [doi:10.1063/1.3373404]

\section{INTRODUCTION}

Microscopic particles exhibit Brownian motion in the liquid dispersion due to their random encounters with the solvent molecules in thermal motion. Diffusion behavior of tiny solute particles in the liquid dispersion has enormous importance in various fields of fundamental science and technology. The diffusion coefficient of the particles in ambient liquid is described by the hydrodynamic theory which assumes that particles move through a homogeneous continuum. For spherical particles dispersed in a stationary liquid, the diffusion coefficient is expressed by the EinsteinStokes relation,

$$
D=\frac{k_{\mathrm{B}} T}{3 \pi \eta d_{\mathrm{H}}}
$$

where $D$ is diffusion coefficient, $k_{\mathrm{B}}$ is Boltzmann constant, $T$ is absolute temperature, $\eta$ is viscosity of the medium, and $d_{\mathrm{H}}$ is hydrodynamic diameter of the particles. Einstein-Stokes relation predicts that the diffusion coefficient of the particles in the homogeneous medium is proportional to $T \eta^{-1}$. This prediction works well in the liquid solvent. Therefore the transport phenomenon of microscopic particles in the liquid

${ }^{a)}$ Electronic mail: sghosh@riken.jp. FAX: +81-48-467-9389. medium is well understood. However, the transport phenomenon of the particles in supercritical fluids (SCFs), which have attracted enormous scientific interest as novel solvents due to their intriguing physicochemical properties, still remains unexplored.

SCFs have attracted immense scientific interest as novel solvents because of their unique advantages over conventional solvents in controlling chemical reactions, extraction processes, as well as in synthesizing and assembling nanoparticles. ${ }^{1-13}$ Their physicochemical properties such as viscosity $(\eta)$, dielectric constant $(\varepsilon)$, and ion product exhibit dramatic changes with respect to temperature $(T)$ and pressure $(P)$. SCFs are considered to be inhomogeneous and perturbed due to the intense density fluctuations and temporary clustering of the molecules both in space and in time. ${ }^{14,15}$ Local fluid structure can be changed from the dense liquidlike structure to the gaslike structure across the ridge of density fluctuations (Fig. 1) by varying temperature or pressure. ${ }^{16-18}$ The ridge corresponds to extrema of the various physical quantities of SCFs which are related to the second derivatives of the Gibbs free energy; for example, heat capacity, isothermal compressibility, ${ }^{19}$ partial molar volumes, ${ }^{1,20,21}$ sound velocity, ${ }^{22}$ and thermal conductivity. ${ }^{23}$ The dynamic properties of SCFs such as the correlation time of density fluctuations, correlation length, and thermal diffu- 


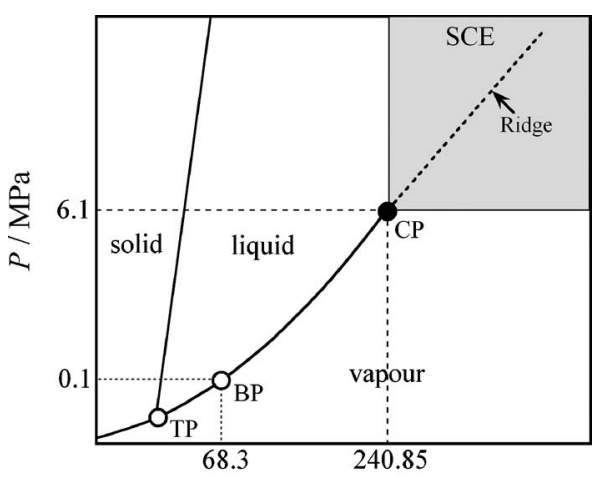

(a)

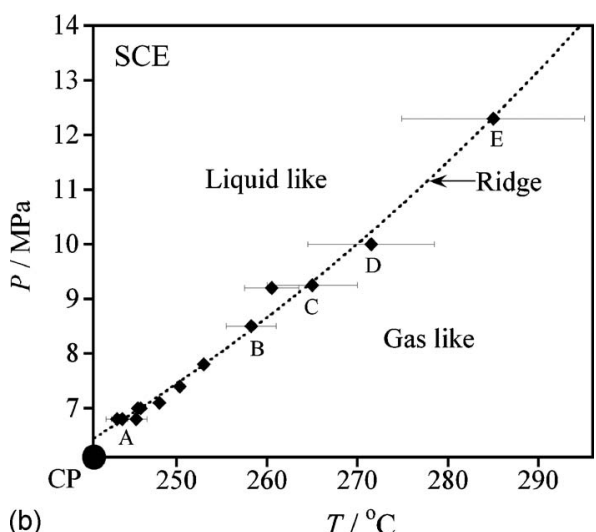

(b)

$T /{ }^{\circ} \mathrm{C}$

FIG. 1. (a) Schematic phase diagram of ethanol. TP, BP, and CP are the triple point, the boiling point, and the critical point, respectively. The gray region above $\mathrm{CP}$ represents SCE. The broken line within SCE is the ridge of density fluctuations. (b) Phase diagram of pure SCE. CP corresponds to the critical point (i.e., $T_{c}=240.85{ }^{\circ} \mathrm{C}$ and $P_{c}=6.1 \mathrm{MPa}$ ). The symbol $(\checkmark)$ corresponds to the thermodynamic conditions $(T, P)$ where DLS intensity was observed maximum. The broken line is the observed ridge of density fluctuations. The error bar at A represents experimental uncertainty. Error bars at B-E show the width of the ridge.

sivity are highly sensitive to temperature and pressure and they show extrema in the vicinity of the critical density. ${ }^{24}$ In a dilute supercritical mixture the solute-solvent interaction gives rise to a cooperative phenomenon that exhibits either a solvent-rich region or a solvent-deficient region around the solute which determines the solubility of the fluid. ${ }^{20,21,25,26}$

We consider that the transport phenomena of nanoparticles in SCFs should be intriguing because the diffusion coefficient of the particles can be manipulated continuously within a wide range in terms of the structural, dynamical, and physicochemical properties of the solvent by varying only the thermodynamic parameters without any additives.

The challenging study of the diffusion behavior of nanoparticles in high temperature and high pressure liquid was initiated by Alargova et al. ${ }^{27}$ in the beginning of this century with the development of a state of the art light scattering system. This pioneering initial work was intriguing which motivated us to explore diffusion behavior of particles in high temperature and high pressure liquids in details both by experiments and theory. In the recent years, a number of studies have been carried out to elucidate the transport phenomena of nanoparticles in high temperature and high pressure water both by experiment and theory. ${ }^{28-32}$ Numerous attempts to investigate the transport phenomenon of nanopar- ticles in supercritical water (SCW) were unsuccessful because particles generally tend to aggregate in high temperature and high pressure water. Our investigations suggest that nanoparticles aggregate in high temperature and high pressure water due to the decrease in the dielectric constant with increasing temperature ${ }^{29}$ and due to the increase in the ionic dissociation of water molecules. ${ }^{32,33}$ It was also suggested that the change in the dielectric constant might have a role to change the surface charge density of the particles. ${ }^{33}$ These effects lower the interparticle electrostatic repulsive potential significantly in high temperature and high pressure water and enhance aggregation of particles.

To investigate the transport behavior of nanoparticles in a SCF one requires an appropriate probe particle which should remain stable against aggregation (or dissolution) in that particular SCF for a reasonably long period of time. After many trials with different solutes and solvents, we found that silica nanoparticles remain stable in supercritical ethanol (SCE) for a long period of time. ${ }^{34}$ Therefore, silica nanoparticle should be an appropriate probe to investigate the diffusion behavior in SCE.

The transport properties of the solutes strongly depend on the viscosity of the solvent. At ambient temperature the viscosities of gases and liquids differ by about two orders of magnitude. In case of ethanol, there is a wide range of intermediate density between 0.4 and $0.2 \mathrm{~g} \mathrm{~cm}^{-3}$, in which $\eta$ depends only weakly on temperature and density (Fig. 2). Within this intermediate density regime, particularly in the vicinity of the critical density, $\eta$ amounts only about onethirtieth $(\sim 3 \%)$ of its value at ambient temperature. The low viscosity and high fluidity property of the SCF results in the high molecular mobilities which should enhance mass diffusion and accelerate the diffusion controlled chemical reactions. However, these are only speculations and no experimental evidences are so far available in the literature.

In this article, we are interested in investigating the diffusion behavior of nanoparticles in SCE within the intermediate density regime, particularly across the ridge of density fluctuations, to elucidate the transport behavior in terms of the structures, dynamics, and physicochemical properties of the solvent.

\section{EXPERIMENTAL}

\section{A. Materials and sample preparations}

Aqueous dispersions of silica nanoparticles (5.13\% solids) and ethanol (99.5\% pure) were purchased from Polysciences, Inc. (Warrington, PA) and Wako Pure Chemical Industries, Ltd. (Osaka, Japan), respectively. They were used as purchased. The working solutions were prepared by mixing the initial dispersion of silica nanoparticles with ethanol. The samples were sufficiently diluted to avoid multiple scattering from the particles. The concentration of the particles in the working solution was $0.04 \mathrm{~g} \mathrm{l}^{-1}$. Concentration of water in the working sample was approximately $0.8 \mathrm{~g} \mathrm{l}^{-1}$.

\section{B. Characterization of the sample}

The size of the particles was measured by dynamic light scattering (DLS) at ambient temperature. The mean diameter 

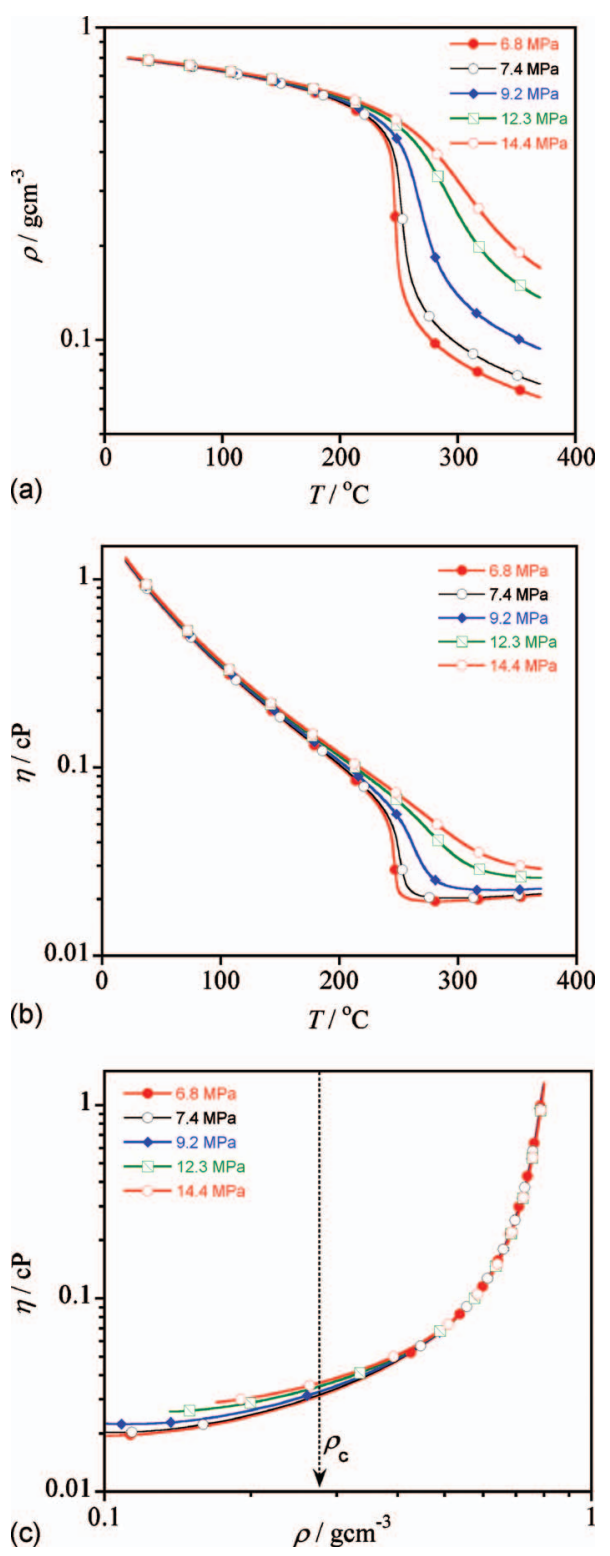

FIG. 2. Properties of ethanol. Temperature dependence of (a) density (Ref. 37) and (b) viscosity (Ref. 40) at pressures of 6.8, 7.4, 9.2, 2.3, and 14.4 MPa. (c) Viscosity with respect to density.

of the particles was $107.9 \mathrm{~nm}$. The $\zeta$ potential of the particles in ethanol dispersion was measured by an electrophoretic light scattering photometer (ELS-Z, Otsuka Electronics Co., Ltd., Osaka, Japan) under atmospheric pressure. The measured $\zeta$ potential was $-56.6 \mathrm{mV}$ at $25^{\circ} \mathrm{C}$. Stability of silica nanoparticles in ethanol against temperature and pressure was examined by heating the working sample at different temperatures (in the range of $265-335^{\circ} \mathrm{C}$ ) for $2 \mathrm{~h}$. The size of the particles remains unchanged and the solubility of silica nanoparticles was found to be negligible within the temperature range of $265-304{ }^{\circ} \mathrm{C}$ under high $(>12 \mathrm{MPa})$ pressure.

\section{Dynamic light scattering measurements}

Diffusion coefficient of the particles in ethanol dispersion was measured by DLS using DLS-820 (Otsuka Electronics Co., Ltd., Osaka, Japan) equipped with a $\mathrm{He}-\mathrm{Ne}$
$(10 \mathrm{~mW}, 632.8 \mathrm{~nm})$ and a solid-state $(50 \mathrm{~mW}, 532 \mathrm{~nm})$ laser. The scattered light was detected by a photomultiplier at a fixed $90^{\circ}$ angle.

The present experimental setup allows the DLS measurements within the temperature limit of $450{ }^{\circ} \mathrm{C}$ and the pressure limit of $40 \mathrm{MPa}$. Diffusion coefficient $(D)$ of the particles was measured within the temperature range of $20-348{ }^{\circ} \mathrm{C}$. For each measurement (at each $T$ ) we have used the freshly prepared sample. During the measurement $T$ and $P$ were controlled with the accuracy of $\pm 0.1{ }^{\circ} \mathrm{C}$ and \pm 0.02 $\mathrm{MPa}$, respectively.

DLS-820 works in a homodyne mode of operation and the scattered light intensity autocorrelation function (ACF) $g^{(2)}(\tau)$ is given by ${ }^{35}$

$$
g^{(2)}(\tau)=\frac{\left\langle I_{S}(t) I_{S}(t+\tau)\right\rangle}{\left\langle I_{S}\right\rangle^{2}},
$$

where $I_{s}$ is the intensity of the scattered light. Corresponding electric field ACF $g^{(1)}(\tau)$ is related to $g^{(2)}(\tau)$ by ${ }^{35}$

$$
g^{(2)}(\tau)=1+\left|g^{(1)}(\tau)\right|^{2} .
$$

For a dispersion of spherical particles with uniform size, $g^{(1)}(\tau)$ is an exponential function of diffusion coefficient of the particles and is expressed by ${ }^{35}$

$$
g^{(1)}(\tau)=\exp \left(-D q^{2} \tau\right),
$$

where $q=\left(4 \pi n / \lambda_{0}\right) \sin (\theta / 2)$ is the scattering vector; $n, \lambda_{0}$, and $\theta$ are refractive index of the medium, wavelength of the incident light, and the scattering angle, respectively.

To measure the temperature and the pressure dependence of $D$ by DLS, $q$ should be calculated at each temperature and pressure using appropriate refractive index of the solvent. Due to the lack of the experimental data in the literature, refractive index of ethanol was calculated by LorentzLorenz equation, which is expressed by ${ }^{36}$

$$
\frac{n^{2}-1}{n^{2}+2}=\frac{4 \pi \rho_{\mathrm{N}} \alpha}{3} \text {. }
$$

Here, $\rho_{\mathrm{N}}$ is the number density and $\alpha$ is the polarizability volume. For ethanol, $\alpha=5.41 \times 10^{-24} \mathrm{~cm}^{3}{ }^{36}$ To calculate the temperature and the pressure dependencies of $n$ from Eq. (5), density of ethanol was computed by the formulation given in Ref. 37. The temperature and the pressure dependencies of the refractive index of ethanol calculated by Eq. (5) are given in the supplementary information. ${ }^{38}$

In order to avoid interparticle interference and multiple scattering we used very dilute samples in which the concentration of the particles was very low.

\section{Dynamic light scattering in supercritical fluids}

Reliability of the measurement of diffusion coefficient of the particles in high temperature and high pressure liquid by DLS experiments was carefully examined using standard solvent and solute. ${ }^{27,28}$ We chose water as a standard solvent for this investigation because the temperature and the pressure dependencies of the viscosity and the refractive index of water, which are required for DLS experiment, are precisely known. We measured the intensity $\mathrm{ACF} g^{(2)}(\tau)$ both from 
pure water and from aqueous colloidal dispersions at different thermodynamic conditions $\left(T_{i}, P_{i}\right)$ within the SCF state. We observed that two distinct diffusive modes exist in observed $g^{(2)}(\tau)$ : one arises from the solvent density fluctuations and the other one arises from the particle diffusion in the solvent. ${ }^{28}$ Results reveal that the relaxation time of the solvent density fluctuations is much slower (millisecond order) than that of the particle diffusion (microsecond order). Results demonstrate that the particle diffusion takes place within a time period which is shorter than the required time period separating two neighboring correlation channels $\left(g^{(2)}(\tau)\right)$ due to solvent density fluctuations. Therefore it is possible to extract the ACF of particle diffusion from the dynamics of solvent density fluctuations by means of DLS experiments.

The intensity ACF $g^{(2)}(\tau)$ is measured in the homodyne regime and corresponding electric field $\mathrm{ACF} g^{(1)}(\tau)$ is calculated to determine the diffusion coefficient of the particles by means of cumulant method. ${ }^{39}$

\section{RESULTS AND DISCUSSIONS}

\section{A. Temperature and pressure dependencies of diffusion coefficient}

The intensity ACF $g^{(2)}(\tau)$ due to the diffusion of silica nanoparticles in ethanol was measured by DLS at several temperatures and pressures within the temperature range of $20-348{ }^{\circ} \mathrm{C}$ and the pressure range of $6.8-14.4 \mathrm{MPa}$. Diffusion coefficients were determined from measured $g^{(2)}(\tau)$ by Eqs. (3) and (4) and by applying cumulant method. ${ }^{39}$ Details of the measurements are reported earlier in Refs. 27 and 28. Measured diffusion coefficients are shown in Fig. 3. Solid lines shown in the figure are the theoretical predictions $\left(D_{\mathrm{th}}\right)$ calculated by the hydrodynamic theory [Eq. (1)]. To calculate $D_{\text {th }}, \eta$ was computed by the formulation given in Ref. 40, which is shown in Fig. 2(b). Results demonstrate that as temperature increases from room temperature at a constant pressure, (i) $D$ increases slowly within the temperature regime $20-240{ }^{\circ} \mathrm{C}$, (ii) $D$ increases very fast at above $240{ }^{\circ} \mathrm{C}$, and (iii) finally $D$ decreases at high temperature within the SCF state. The temperature dependencies of $D$ were examined at pressures of $6.8,7.4,9.2,12.3$, and $14.4 \mathrm{MPa}$. Observed results demonstrate that $D$ starts to decrease at a characteristic temperature which depends on applied pressure.

Results reveal that the diffusion behavior of nanoparticles in the supercritical state is highly sensitive to temperature and pressure. When $T$ is increased at a constant pressure $P_{i}$ (for example, 6.8, 7.4, 9.2, 12.3, and $14.4 \mathrm{MPa}$ ), $D$ increases and reaches its maximum $\left(D^{\max }\right)$ at the characteristic temperature $\left(T_{i}\right)$ within the SCF state. It is observed that $T_{i}$ is $521 \mathrm{~K}$ for $6.8 \mathrm{MPa}, 530 \mathrm{~K}$ for $7.4 \mathrm{MPa}, 551 \mathrm{~K}$ for $9.2 \mathrm{MPa}$, $577 \mathrm{~K}$ for $12.3 \mathrm{MPa}$, and $588 \mathrm{~K}$ for $14.4 \mathrm{MPa}$ (Fig. 3). Results clearly demonstrate that $D$ decreases abruptly when temperature is increased above $T_{i}$ at pressure $P_{i}$.

Observed results suggest that the diffusion coefficient reaches $D^{\max }$ in the SCF state at a particular thermodynamic condition $\left(T_{i}, P_{i}\right)$, which is expressed by the empirical formula,
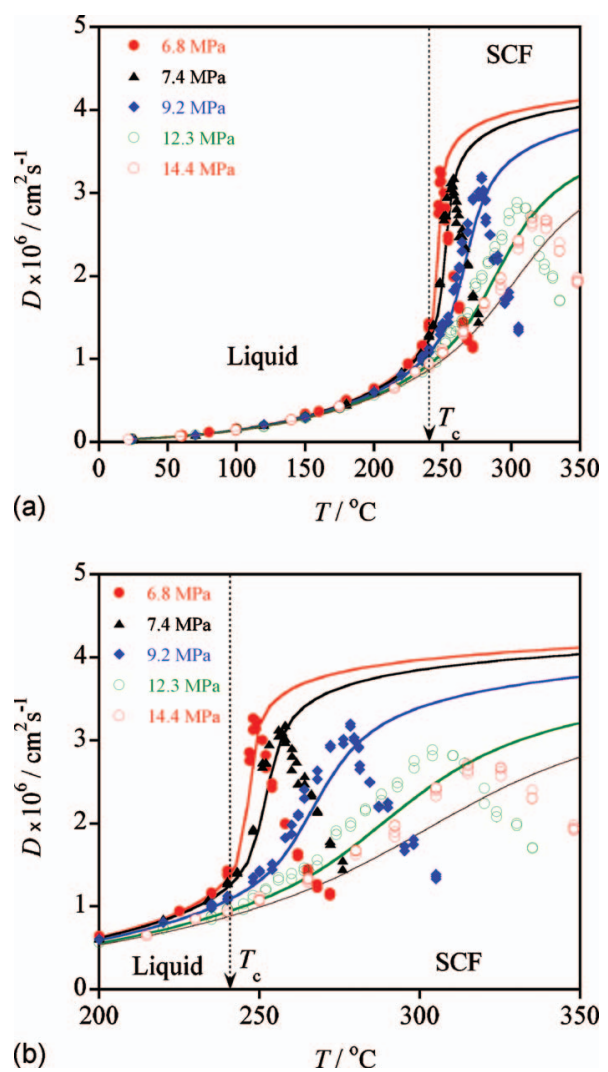

FIG. 3. (a) Diffusion coefficient of silica nanoparticles in ethanol as a function of temperature at 6.8, 7.4, 9.2, 12.3 (Ref. 34), and 14.4 MPa. Solid lines represent Einstein-Stokes relationship [Eq. (1)]. (b) Part of figure (a) is enlarged.

$$
T_{r i}=\beta P_{r i}^{\nu} \quad\left(\text { for } T_{r i}>1, P_{r i}>1\right) .
$$

Here, $T_{r i}=T_{i} / T_{c}$ is reduced temperature and $P_{r i}=P_{i} / P_{c}$ is reduced pressure. $T_{c}$ and $P_{c}$ are the critical temperature and the critical pressure, respectively. $\beta$ and $\nu$ are constants. For ethanol $T_{c}=514 \mathrm{~K}$ (Ref. 41 ) and $P_{c}=6.1 \mathrm{MPa}^{41}$ The empirical formula [Eq. (6)] is described in Fig. 4 by the solid line. From the fitting with the experimental results we find that $\beta \cong 1.0$ and $\nu \cong 0.16$. The solid line in Fig. 4 describes the thermodynamic state of ethanol which facilitates the fast-

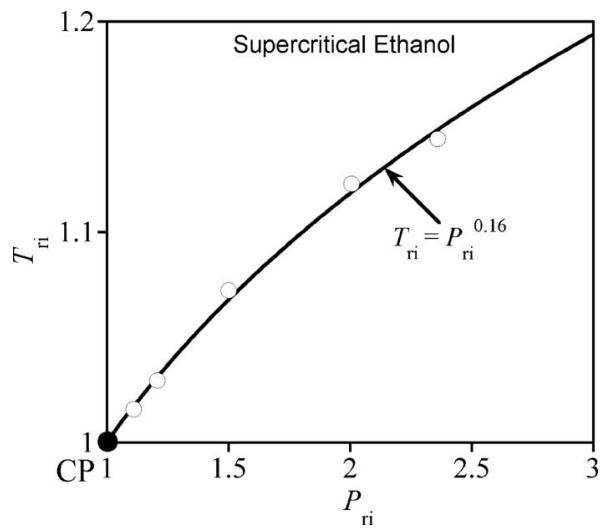

FIG. 4. Reduced temperature $T_{r i}$ as a function of reduced pressure $P_{r i}$. The open circles represent the thermodynamic condition where $D^{\max }$ was observed. The solid line represents the empirical formula [Eq. (6)], which describes the thermodynamic condition for the fastest diffusion coefficient in SCE. The solid circle is the critical point of ethanol. 
est diffusion of solute particles. The empirical formula might have an upper limit for temperature and pressure, which we did not observe within our experimental limit.

We would like to note that when a trace amount of solute dispersion is added into pure ethanol the working solution is no longer pure, and therefore the critical constants should shift a little. The working solution used for our experiment contains trace amount of water which is estimated to be $0.8 \mathrm{~g} \mathrm{l}^{-1}$. A majority of the published values for the critical temperature for ethanol lie between 513.9 and $516.7 \mathrm{~K}$ and the critical pressure lies between 6.1 and $6.4 \mathrm{MPa}^{42}$ Our experiments demonstrated that the critical constants of the working solution lie within this limit given in the literature. Therefore, no further correction was done.

Experimental result is analyzed by the hydrodynamic theory which assumes that the solute particles move through a homogeneous liquid continuum. The comparison between the experimental observations and the theoretical predictions suggests that the hydrodynamic theory works well within the liquid phase of ethanol which corresponds to the temperature regime of $20-240{ }^{\circ} \mathrm{C}$ at pressures $P \geq 6.8 \mathrm{MPa}$ (Fig. 3). In the SCF state, however, $D$ deviates from the theoretical predictions $\left(D_{\text {th }}\right)$. Results show that at $12.3 \mathrm{MPa}$ pressure, $D$ is larger than $D_{\text {th }}$ within the temperature range, $241-304{ }^{\circ} \mathrm{C}$, whereas it decreases abruptly at $T>304{ }^{\circ} \mathrm{C}$. Similar behavior was observed for pressures of 6.8, 7.4, 9.2, and 14.4 MPa. We conclude that at pressure $P_{i}, D$ is appreciably larger than $D_{\text {th }}$ within the temperature range, $T_{c}<T<T_{i}$, whereas $D$ deviates abruptly downward from $D_{\text {th }}$ at $T>T_{i}$.

Results suggest that the diffusion coefficient of particles in the SCF state (within the thermodynamic range, $T_{c}<T$ $<T_{i}$ ) is larger than what is predicted by the hydrodynamic theory. We observed that the difference between the theoretical prediction $\left(D_{\mathrm{th}}\right)$ and the experimental $D$ depends on applied pressure. We estimated that in the vicinity of $D_{\max }$ the differences between $D$ and $D_{\text {th }}$ are about $13 \%$ for $9.2 \mathrm{MPa}$, $14.9 \%$ for $12.3 \mathrm{MPa}$, and $20 \%$ for $14.4 \mathrm{MPa}$.

Here, we make an error analysis to estimate the uncertainties involved in the predicted diffusion coefficient. $D_{\text {th }}$ might involve an uncertainty which arises from the uncertainty in the viscosity data. ${ }^{40}$ The computed viscosity used in calculating $D_{\text {th }}$ reproduces experimental viscosity data ${ }^{43}$ within an accuracy of $0.7 \%-2.1 \%$. However, the experimental data ${ }^{43}$ taken from the literature have uncertainties as large as $\pm 6 \%$ in the supercritical region. We estimate that the uncertainty in $D_{\text {th }}$ might be as large as $\pm 8.1 \%$ in the supercritical regime. This suggests that the significant differences between observed $D$ and $D_{\text {th }}$ (Fig. 3) do not arise from the uncertainties in viscosity data. Finally, we conclude that the observed diffusion coefficient of nanoparticles in supercritical solvent is larger than what is predicted by the hydrodynamic theory.

To measure $D$ by DLS one also requires the refractive index of the solvent at each temperature and pressure. We calculated refractive index of ethanol by Eq. (5) because no refractive index data for SCE are available in the literature. To calculate refractive index by Eq. (5), we computed density of ethanol by the formulation given in Ref. 37. These density data have an accuracy within $\pm 0.2 \%$. This formula- tion is based on the experimental data ${ }^{44}$ which is estimated to be accurate within $\pm 0.4 \%$ in the supercritical state. We consider that these trivial uncertainties in the refractive index would not affect our results.

We observed that the uncertainties at critical point and very close to the critical point are large and unpredictable. Therefore, we excluded experimental data at critical point and very close to the critical point.

Results suggest that the diffusion coefficient of nanoparticles in the supercritical solvent is appreciably larger than what is predicted by the hydrodynamic theory, and we find that a special thermodynamic state exists in the supercritical solvent where nanoparticles move with the fastest diffusion coefficient. This diffusion behavior is highly intriguing and unique.

To elucidate the diffusion behavior of nanoparticles in the SCF in terms of the solvent structures we analyze observed results as a function of the density and the viscosity of the solvent. The density and the viscosity were varied by changing temperature and pressure. Figure 5(a) shows that $D$ increases as the density of the solvent decreases, $D$ reaches the maximum $\left(D^{\max }\right)$ at a characteristic density, and finally $D$ decreases abruptly from its maximum at very low density gaslike solvent. Solid lines represent theoretical predictions $\left(D_{\mathrm{th}}\right)$ at different pressures. Predictions by the hydrodynamic theory agree well with the experimental results within the higher density $\left(0.8-0.4 \mathrm{~g} \mathrm{~cm}^{-3}\right)$ liquid medium. Results reveal that $D$ is significantly larger than the predictions $\left(D_{\mathrm{th}}\right)$ in the intermediate density $\left(0.4-0.2 \mathrm{~g} \mathrm{~cm}^{-3}\right)$ regime, and $D$ reaches the maximum $\left(D^{\max }\right)$ when the density of the solvent is slightly less than the critical density $\left(\rho_{c}=0.276 \mathrm{~g} \mathrm{~cm}^{-3}\right){ }^{37}$ The changes in the viscosity with respect to the density of the solvent are shown in Fig. 5(b). Observed $D^{\max }$ at different applied pressures and corresponding solvent densities are shown in Fig. 5(c). Results show that $D^{\max }$ is larger at lower fluid density. However, $D$ drops abruptly at very low fluid density.

Results reveal two important facts. In the low density $\left(\rho<\rho_{c}\right)$ gaslike solvent where the change in viscosity with respect to pressure is trivial, we observe that (i) for a constant density, $D$ changes significantly with little pressure change [Fig. 5(a)], and (ii) $D^{\max }$ changes significantly with respect to solvent density [Fig. 5(c)]. Results suggest that the diffusion behavior of the particles in the gaslike SCF is almost independent of the viscosity of the solvent.

Diffusion behavior of nanoparticles in the SCFs in terms of solvent properties is an unknown issue to be explored. No relevant information is available in the literature so far. It might be interesting to note that Butenhoff and co-workers ${ }^{45}$ examined the binary diffusion coefficient $\left(D_{12}\right)$ of $\mathrm{NaNO}_{3}$ molecules in SCW. They observed a "critical slowing down" in the vicinity of the critical point of water. Farther from the critical point, however, they observed that $D_{12}$ is about 15 times larger than its value at ambient temperature. Although the size of a silica nanoparticle (examined in this work) is approximately 200 times larger than the size of a $\mathrm{NaNO}_{3}$ molecule, we interestingly find that the diffusion coefficient of a silica nanoparticle in SCE at maximum $\left(D^{\max }\right)$ is about 100 times larger than its value at ambient condition. A com- 

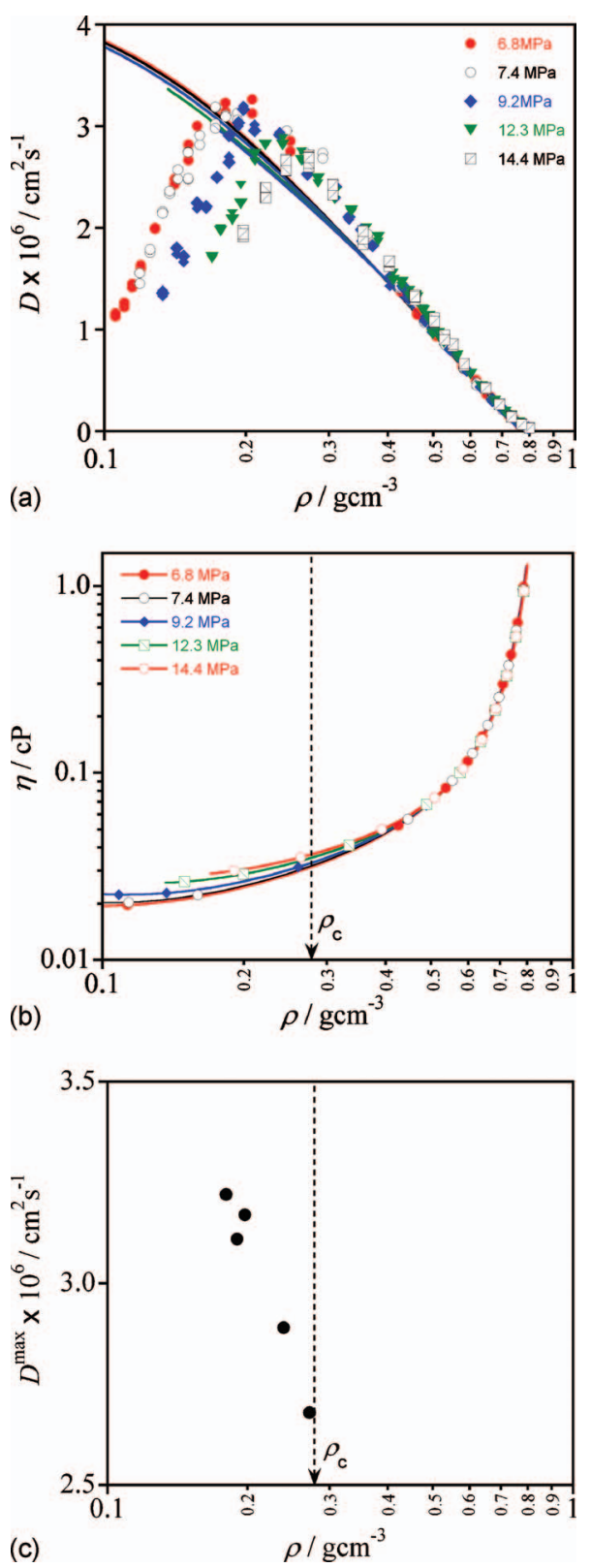

FIG. 5. (a) Diffusion coefficient with respect to the density of ethanol at different pressures. Solid lines are the predictions by the hydrodynamic theory [Eq. (1)]. (b) Figure 2(c) is replotted to show the corresponding fluid viscosity. (c) $D^{\max }$ with respect to solvent density.

parison between these results indicates that the diffusion coefficient of a molecule or a particle in the SCF may depend on solute-solvent interaction.

The observed diffusion behavior of nanoparticles in the gaslike SCF is a novel finding. We consider that the diffusion behavior of nanoparticles in SCF is related to the solvent structures, solvent-solvent, and solute-solvent interactions. This will be discussed in section $\mathrm{C}$.

\section{B. Kinetic stability of the particles in high temperature and high pressure solution}

Dispersion stability of nanoparticles or colloids in the liquid is governed by the net forces acting between the surfaces of the particles. The net interparticle potential in a liquid is generally accounted for by Derjaguin-Landau-VerweyOverbeek (DLVO) theory, ${ }^{46-51}$

$$
V_{\mathrm{D}}=V_{\mathrm{A}}+V_{\mathrm{R}}
$$

where $V_{\mathrm{D}}$ is net DLVO potential, $V_{\mathrm{A}}$ is van der Waals attractive potential, and $V_{\mathrm{R}}$ is the electrostatic repulsive potential. For two identical spherical particles of radius $r$ and separated by a distance $H, V_{\mathrm{A}}$ is expressed by ${ }^{49}$

$$
V_{\mathrm{A}}=-\frac{A}{12}\left[\frac{1}{x(x+2)}+\frac{1}{(x+1)^{2}}+2 \ln \left(\frac{x(x+2)}{(x+1)^{2}}\right)\right] \text {. }
$$

Here, $A$ is the Hamaker's constant, ${ }^{52}$

$$
A=\frac{3}{4} k_{\mathrm{B}} T\left(\frac{\varepsilon-\varepsilon_{1}}{\varepsilon+\varepsilon_{1}}\right)^{2}+\frac{3 h \nu_{e}}{16 \sqrt{2}} \frac{\left(n^{2}-n_{1}^{2}\right)^{2}}{\left(n^{2}+n_{1}^{2}\right)^{3 / 2}},
$$

$x=H / 2 r, \varepsilon$ is the dielectric constant of the solvent, $\varepsilon_{1}$ is the dielectric constant of the solute, $h$ is the Planck's constant, $\nu_{e}$ is the electronic absorption frequency, and $n_{1}$ is the refractive index of the particles.

In a low dielectric solvent the ion concentration is very low and the Debye screening length is very large. For such a system, the effect of Debye screening length is negligible and $V_{\mathrm{R}}$ is approximated as ${ }^{53}$

$$
V_{R}=\frac{4 \pi \varepsilon \varepsilon_{0} r^{2} \zeta^{2}}{R}
$$

where $R$ is the distance between the centers of the particles, $\varepsilon_{0}$ is the permittivity of the vacuum, and $\zeta$ is zeta potential.

The stability of the particles in the solution depends on both the surface properties of the particles and the physicochemical properties of the solvent. Temperature dependence of the net DLVO potential is numerically calculated by Eq. (7) considering that the properties of the solvent (such as $\varepsilon$, $\eta$, and $n$ ) change with temperature and pressure but those of the solutes (such as $\zeta, \varepsilon_{1}$, and $n_{1}$ ) remain unchanged. Due to these assumptions, temperature dependence of the stability of particles in the dispersion is solely determined by the temperature and the pressure dependence of the properties of the solvent. The physical quantities used in this calculation are given in Table I. Due to the limited dielectric constant data for ethanol in the literature, we have calculated the DLVO potential barrier only at $14.4 \mathrm{MPa}$.

The net DLVO potential $\left(V_{\mathrm{D}}\right)$ and the potential barrier at maximum $\left(V_{\mathrm{D}}^{\mathrm{max}}\right)$ are numerically calculated at different tem-

TABLE I. Physical quantities used for the numerical calculations. Mean hydrodynamic radius $(r)$ is measured at $T=25{ }^{\circ} \mathrm{C}$. $\zeta, n_{1}$, and $\varepsilon_{1}$ are the zeta potential, refractive index, and dielectric constant of silica nanoparticles, respectively.

\begin{tabular}{lccccc}
\hline \hline Materials & $r(\mathrm{~nm})$ & $\zeta(\mathrm{mV})$ & $n_{1}$ & $\varepsilon_{1}$ & $\nu_{e} / 10^{15}\left(\mathrm{~s}^{-1}\right)$ \\
\hline Silica nanoparticle & 53.95 & -56.6 & $1.544^{\mathrm{a}}$ & $4.5^{\mathrm{b}}$ & 2.65 \\
\hline \hline
\end{tabular}




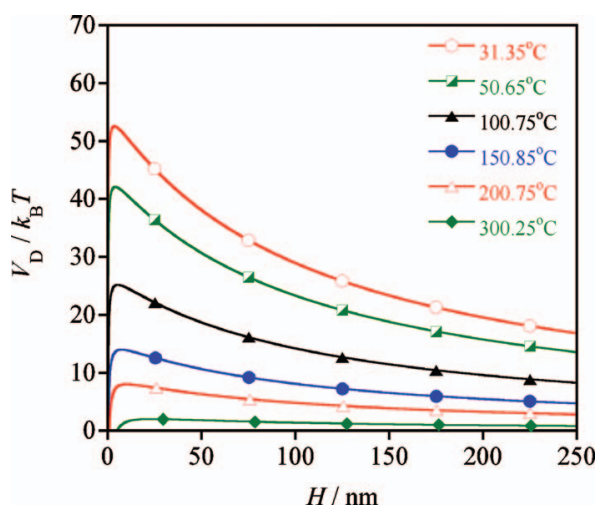

FIG. 6. DLVO potential with respect to the distance between the surfaces of the particles at $P=14.4 \mathrm{MPa}$. Numerical calculation is carried out by Eq. (7) (Ref. 34).

peratures for $P=14.4 \mathrm{MPa}$. Numerical results are described in Fig. 6 and 7. Numerical calculations show that $V_{\mathrm{D}}^{\max }$ decreases from $53 k_{\mathrm{B}} T$ to $2 k_{\mathrm{B}} T$ when temperature is increased from 30 to $300{ }^{\circ} \mathrm{C}$.

Colloidal dispersions are thermodynamically unstable, and they are stabilized kinetically. The aggregation rate of the particles in the solution depends on (i) the collision frequency with which the particles encounter each other and (ii) the probability that their thermal energy is sufficiently high to overcome the kinetic potential barrier. In the presence of a repulsive potential barrier, only a fraction of the collisions result in the permanent aggregations. The complete aggregation kinetics can be calculated if it is assumed that the rate constants are practically independent of particle size. The universal rate constant for diffusion-controlled aggregation of identical spherical particles is expressed by ${ }^{51}$

$$
k_{r} \cong \frac{8 k_{\mathrm{B}} T}{3 \eta}
$$

Here, $k_{r}$ is independent of the particle size and the collision frequency with which the particles encounter one another is expressed by

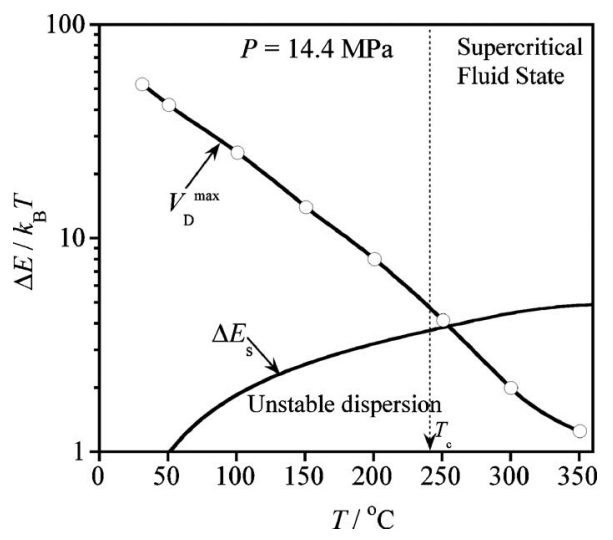

FIG. 7. DLVO potential barrier $\left(V_{D}^{\max }\right)$ between two silica nanoparticles. $V_{D}^{\max }$ is estimated from $V_{D}$ (Fig. 6) (Ref. 34). $\Delta E_{s}$ is calculated for $n_{c}$ $=2.36 \times 10^{13} / \mathrm{m}^{3}, t=7200 \mathrm{~s}$, and $P=14.4 \mathrm{MPa}$ by Eq. (15).

$$
f_{c}=k_{r} n_{c}=\frac{8 k_{\mathrm{B}} T}{3 \eta} n_{c},
$$

where $n_{c}$ is the number of the particles per unit volume. To stabilize particles in the solution at $T$ for a period of time $t$, one requires that the probability of occurring a permanent aggregation of two colliding particles overcoming their kinetic potential energy barrier $(\Delta E)$ should be kept less than $1 / f_{c} t$, i.e.,

$$
P_{\text {agg }}(T, t)=\exp \left(-\Delta E / k_{\mathrm{B}} T\right)<\frac{1}{f_{c} t},
$$

where $P_{\mathrm{agg}}(T, t)$ is the probability of occurring a permanent aggregation at $T$ within the time period $t$. Eqs. (12) and (13) yield

$$
\frac{\Delta E}{k_{\mathrm{B}} T}>-\ln \left(\frac{3 \eta}{8 k_{\mathrm{B}} T n_{c} t}\right) .
$$

Hence, the minimum kinetic barrier required to stabilize particles in a high temperature and high pressure solution is

$$
\Delta E_{S}=-\ln \left(\frac{3 \eta}{8 k_{\mathrm{B}} T n_{c} t}\right) k_{\mathrm{B}} T .
$$

If the kinetic potential energy barrier $\Delta E$ is much higher than $\Delta E_{S}$, i.e., $\Delta E \gg \Delta E_{S}$, the solution is stable and the particles remain dispersed in the liquid for a long period of time. If $\Delta E \approx \Delta E_{S}$, particles start to aggregate and the aggregation proceeds slowly. If $\Delta E<\Delta E_{S}$, the aggregation will proceed very fast. Equation (15) is a good approximation at the initial stage of the aggregation process. As the aggregation proceeds additional considerations are required. The temperature and the pressure dependence of $\Delta E_{S}$ can be predicted from Eq. (15) by introducing the temperature and the pressure dependence of $\eta$.

\section{Diffusion behavior and fluid properties}

In this section we discuss the implications of the observed diffusion behavior of nanoparticles in SCF. In case of a conventional solvent, which is considered to be homogeneous and unperturbed, the decrease in diffusion coefficient is usually attributed to the increase in particle size or aggregation of the particles or formation of the clusters. However, it is unknown what really occurs in the SCFs. Here we discuss the observed diffusion behavior in SCF in terms of solvent structures and properties.

For the DLS experiments we used a flow-type sample cell. Due to the complex structure of the sample cell, it was impossible for us to recover and investigate the actually measured (heated) samples after the experiment to examine whether particles aggregated or not. Therefore we used an alternative method. We examined the diffusion coefficient of the particles in SCE as a function of heating time $(t)$ at a fixed temperature and pressure for a long time. If particles aggregate diffusion coefficient would decrease with respect to heating time, which is expected due to the consequence of Eq. (15).

We measured $D$ at three different temperatures of 265 , 305, and $325^{\circ} \mathrm{C}$ at $P=12.3 \mathrm{MPa}$. Results are shown in Fig. 


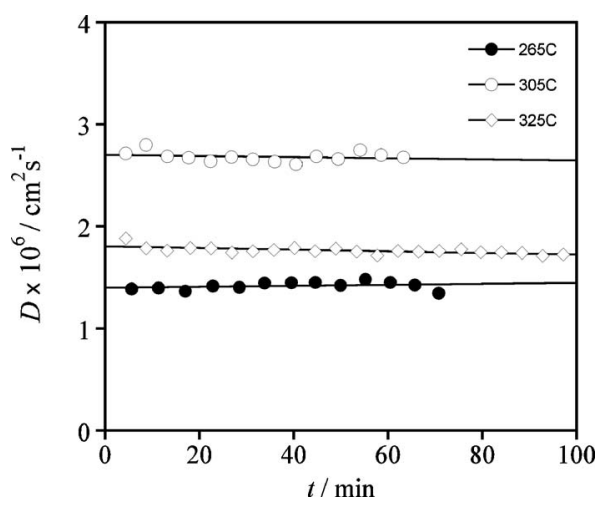

FIG. 8. Diffusion coefficient of silica nanoparticles as a function of time $t$ spent by the particles at temperatures of 265,305 , and $325{ }^{\circ} \mathrm{C}$ under a constant pressure of 12.3 MPa (Ref. 34).

8. We observe that $D$ remains unchanged for a long period of time $(t>3600 \mathrm{~s})$ at all three temperatures. This result demonstrates that size of the particles remains unchanged within the observed time period. Stability criterion [Eq. (15)] suggests that when particles start to aggregate in the high temperature and high pressure solvent the aggregation proceeds fast with heating time due to low interparticle potential barrier and high collision frequency. As a result, size of the aggregates increases and the diffusion coefficient should decrease with respect to heating time. These are demonstrated by experiments shown in Fig. 9. Figure 9(a) shows the diffusion coefficient of colloidal gold $(\mathrm{CG})^{27,29}$ and polystyrene latex (PSL) (Ref. 29) in water as a function of temperature at $25 \mathrm{MPa}$. The solid lines represent diffusion coefficient $\left(D_{\mathrm{th}}\right)$ predicted by the hydrodynamic theory. The abrupt decrease in $D$ occurred in high temperature water due to the aggregation of the particles. ${ }^{27,29}$ Alargova et al. ${ }^{27}$ examined the diffusion coefficient and the hydrodynamic diameter of PSL with respect to heating time $t$ in high temperature and high pressure water. Figure 9(b) shows that $D$ decreases and hydrodynamic diameter increases as the sample is heated at a constant temperature $\left(300{ }^{\circ} \mathrm{C}\right)$ and pressure $(15 \mathrm{MPa})$. Results suggest that once the aggregation starts it continues fast as time passes even though the temperature and pressure remain constant. The stability criterion of the high temperature and high pressure colloidal dispersion is in agreement with this experimental evidence.

From the above discussion and based on the experimental fact (Fig. 8) that the size of silica nanoparticles remains unchanged in SCE for a long period of time, we suggest that aggregation of the particles does not occur and the SCE dispersion remains stable for a long period of time at the given experimental conditions.

Recently, we examined the size of the particles as a function of temperature in SCE by a simple method. ${ }^{34}$ We sealed a part of the fresh sample in a reactor (made of a Ni-based supermetal known as Hastelloy C22), which was then heated to the desired temperature in a heating bath and kept at each temperature for $2 \mathrm{~h}$. After $2 \mathrm{~h}$ of heat treatment the reactor was naturally cooled down to the ambient temperature. The heat treated sample was then collected from the reactor and the size of the particles was measured by DLS at $25^{\circ} \mathrm{C}$. Results showed that particles do not aggregate within the
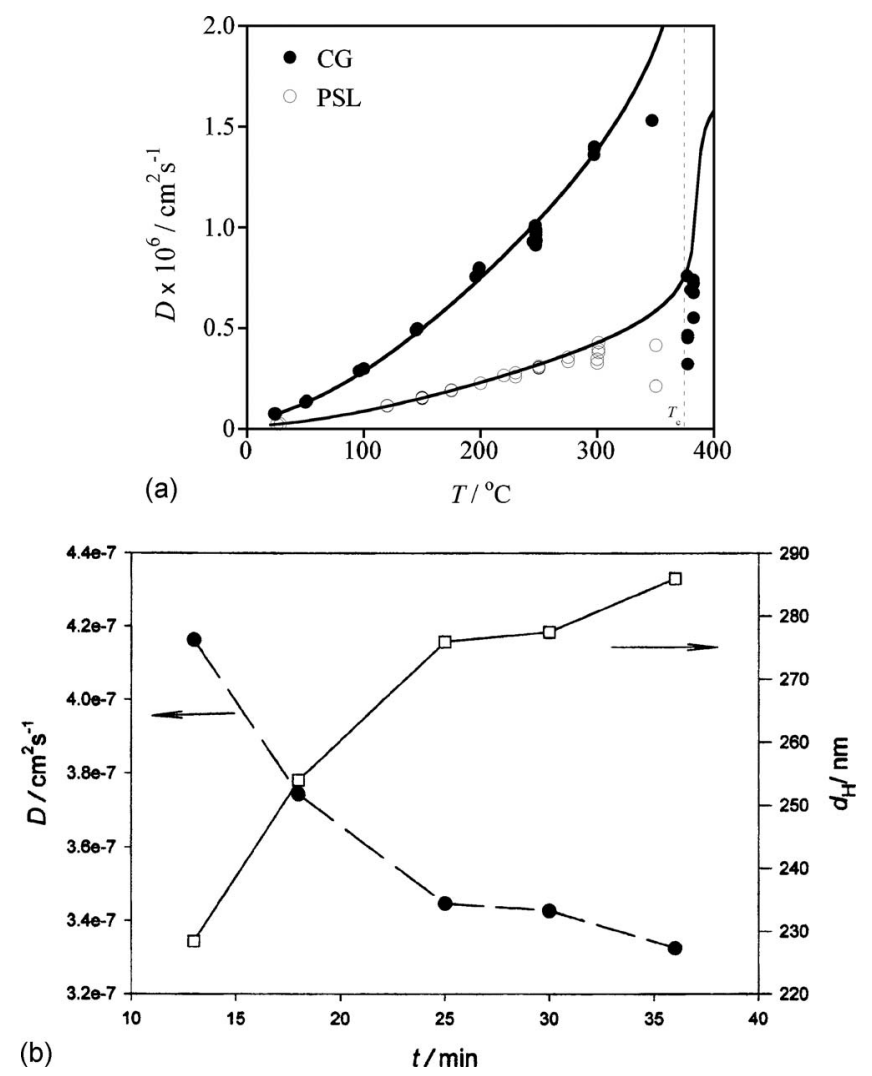

FIG. 9. (a) Diffusion coefficient of CG (Refs. 27 and 29) and PSL (Ref. 29) in water at $P=25 \mathrm{MPa}$. The size of CG is $66 \mathrm{~nm}$ and PSL is $214 \mathrm{~nm}$ in diameter. The solid lines represent theoretical prediction $\left(D_{\mathrm{th}}\right)$. (b) Diffusion coefficient and hydrodynamic diameter of PSL as a function of time $t$ spent by the particles in water at $T=300{ }^{\circ} \mathrm{C}$ and $P=15 \mathrm{MPa}$. Reprinted with permission from R. G. Alargova, S. Deguchi, and K. Tsujii, Colloids Surf., A 183-185, 303 (2001). Copyright 2001, Elsevier Science B.V.

temperature range, $20-304{ }^{\circ} \mathrm{C}$ (at $P=12.3 \mathrm{MPa}$ ); however, they aggregate at temperatures $T \geq 305{ }^{\circ} \mathrm{C} .{ }^{34}$ Based on this simple experiment, we suggested that the decrease in $D$ in DLS experiment at $P=12.3 \mathrm{MPa}$ and $T \geq 305{ }^{\circ} \mathrm{C}$ occurs due to aggregation of the particles. ${ }^{34}$ One must realize that the DLS experiment and this reactor experiment were not performed at the same conditions. The pressure path is very important at high temperature and high pressure experiment. For DLS experiment pressure was controlled and measured at each temperature, but for the heat treatment experiment in the reactor, we measured only the final pressure at each temperature; and the pressure path was not controlled. Since the pressure path was not controlled, these investigations should be reexamined by means of a controlled pressure system, which was not possible at this stage due to technical difficulties.

The diffusion behavior of nanoparticles in the SCF drastically changes with pressure, which has important implications in optimizing the chemical processes. The enhancement of the diffusion coefficient of the particles within the vicinity of the critical density is unique and fascinating. Figure 10 shows the ridge of density fluctuations in SCE (without particles) and the thermodynamic condition for maximum diffusion coefficients of nanoparticles in SCE. Results show that the thermodynamic state for the maximum diffusion coefficient (solid line) lies slightly below the ridge (i.e., within the gaslike $\mathrm{SCF})$. 


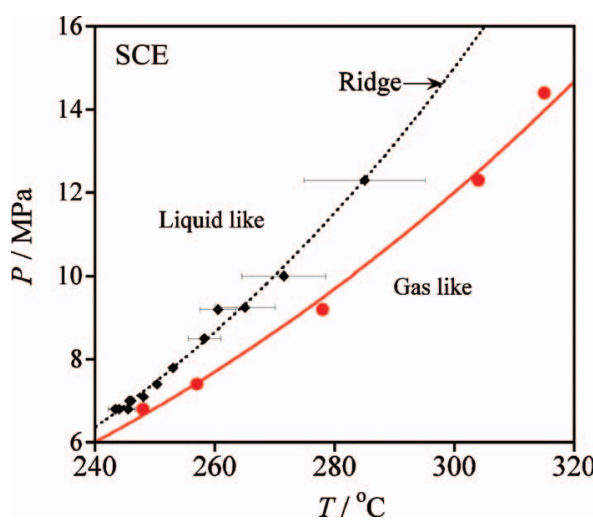

FIG. 10. Phase diagram of SCE. The broken line [same as Fig. 1(b)] represents the ridge of density fluctuations in pure ethanol. Solid circles are the observed thermodynamic condition $(T, P)$ for the fastest diffusion coefficient of silica nanoparticles in SCE. The solid line is an extrapolation for the guide to the eye.

The enhancement of the diffusion coefficient in the vicinity of the critical density and the abrupt decrease in the diffusion coefficient at very low density gaslike fluid may be associated with the rapid change in the direct correlation function (related to the effective intermolecular potential) within the SCF. To explain observed diffusion behavior we consider van der Waals fluid model which gives good phenomenological description for the behavior of the supercritical state and presents the essence of the density fluctuations. ${ }^{16}$ Infinitely dilute supercritical mixtures may be classified into any of the three categories: attractive, weakly attractive, and repulsive, according to the sign of the solute's partial molar properties and of the excess number of solvent molecules surrounding a given solute. ${ }^{21,25,26}$ The attractive or repulsive character of a van der Waals mixture is determined by the ratio of solute to solvent specific energies, referred to the respective molecular volumes. When temperature is increased at a constant pressure, the SCF expands (i.e., density decreases) and the intermolecular (solvent-solvent) potential rapidly changes from attractive to the repulsive across the ridge of density fluctuations. The presence of a trace amount of solute within a supercritical solvent characterized by longranged density fluctuations gives rise to a cooperative phenomenon, ${ }^{21,25,26}$ which, for attractive systems, involves the formation of a large solvent-rich region around each solute particle. Similarly, for repulsive system, the cooperative phenomenon involves the formation of a large solventdeficient region around each solute. The solvent-rich region around a particle occurs in the liquidlike SCF and the solvent-deficient region around a particle occurs in the low density gaslike SCF. We suggest that the unique diffusion behaviors observed in the vicinity of the critical density and within the low density gaslike fluid are associated with the rapid change in the solvent-solvent and solute-solvent interaction potentials upon density change across the ridge of density fluctuations and within the gaslike fluid.

\section{CONCLUSIONS}

Diffusion behaviors of silica nanoparticles have been systematically investigated in SCE in terms of solvent prop- erties by varying temperature and pressure to elucidate how the inhomogeneous solvent structures and the density fluctuations in the solvent affect the transport properties of the particles. Results demonstrate that at a constant pressure $\left(P>P_{c}\right)$ the diffusion coefficient $(D)$ of the particles increases with increasing temperature, reaches its maximum $D^{\max }$, and finally decreases abruptly at very low density gaslike SCF. We observed that the Einstein-Stokes relationship does not work in the SCF state. Results show that in the vicinity of the critical density, $D$ is significantly larger than what is expected by the Einstein-Stokes relationship. $D$ reaches $D^{\max }$ at a particular thermodynamic condition, which is expressed by the empirical equation $T_{r i}=P_{r i}^{0.16}$ (for $T_{r i}>1$, $\left.P_{r i}>1\right)$. Here, $T_{r i}=T_{i} / T_{c}$ and $P_{r i}=P_{i} / P_{c}$; for ethanol, $T_{c}=514 \mathrm{~K}$ and $P_{c}=6.1 \mathrm{MPa}$. Results further reveal that $D$ reaches $D^{\max }$ at a solvent density which is slightly lower than the critical density. This diffusion behavior of the particles in the SCF is novel, unique, and intriguing. We suggest that the enhancement of the diffusion coefficient in the vicinity of the critical density and the abrupt decrease in the diffusion coefficient within the low density gaslike fluid are associated with the change in the solvent-solvent and solute-solvent direct correlation function upon density change across the ridge and within the gaslike fluid.

\section{ACKNOWLEDGMENTS}

Dynamic light scattering (DLS) experiments were carried out at Japan Agency for Marine-Earth Science and Technology (JAMSTEC). Authors gratefully acknowledge the support of Dr. Shigeru Deguchi at JAMSTEC. Dr. Marcia Huber, NIST, has kindly provided us the software to compute the viscosity and the density data. We sincerely acknowledge the continuous support and the encouragement of Professor Koki Horikoshi (JAMSTEC). One of the authors (S.K.G.) acknowledges the support of the Foreign Postdoctoral Researcher (FPR) program of RIKEN.

${ }^{1}$ C. A. Eckert, B. L. Knutson, and P. G. Debenedetti, Nature (London) 383, 313 (1996).

${ }^{2}$ J. D. Holmes, K. P. Johnston, R. C. Doty, and B. A. Korgel, Science 287, 1471 (2000).

${ }^{3}$ R. W. Shaw, T. B. Brill, A. A. Clifford, C. A. Eckert, and E. U. Franck, Chem. Eng. News 69, 26 (1991).

${ }^{4}$ H. Weingärtner and E. U. Franck, Angew. Chem., Int. Ed. 44, 2672 (2005).

${ }^{5}$ P. E. Savage, Chem. Rev. (Washington, D.C.) 99, 603 (1999).

${ }^{6}$ N. Akiya and P. E. Savage, Chem. Rev. (Washington, D.C.) 102, 2725 (2002).

${ }^{7}$ K. P. Johnston and P. S. Shah, Science 303, 482 (2004).

${ }^{8}$ P. S. Shah, T. Hanrath, K. P. Johnston, and B. A. Korgel, J. Phys. Chem. B 108, 9574 (2004).

${ }^{9}$ T. Mousavand, S. Takami, M. Umetsu, S. Ohara, and T. Adschiri, J. Mater. Sci. 41, 1445 (2006).

${ }^{10}$ K. Sue, N. Kakinuma, T. Adschiri, and K. Arai, Ind. Eng. Chem. Res. 43, 2073 (2004).

${ }^{11}$ Y. Hakuta, T. Adschiri, and K. Arai, J. Mater. Sci. Lett. 17, 1211 (1998).

${ }^{12}$ T. Adschiri, T. Mousavand, S. Takami, M. Umetsu, S. Ohara, T. Naka, and T. Tsukada, in Supercritical Hydrothermal Synthesis of Advanced Metal Oxide Nanoparticles in Materials Chemistry in Supercritical Fluid, edited by H. Wakayama (Research Signpost, Kerala, India, 2005).

${ }^{13}$ C. A. Fernandez, E. M. Hoppes, J. G. Bekhazi, C. Wang, R. J. Wiacek, M. G. Warner, G. E. Fryxell, J. T. Bays, and R. S. Addleman, J. Phys. Chem. C 112, 13947 (2008). 
${ }^{14}$ S. C. Tucker and M. W. Maddox, J. Phys. Chem. B 102, 2437 (1998); S. C. Tucker, Chem. Rev. (Washington, D.C.) 99, 391 (1999).

${ }^{15}$ O. Kajimoto, Chem. Rev. (Washington, D.C.) 99, 355 (1999).

${ }^{16}$ K. Nishikawa, K. Kusano, A. A. Arai, and T. Morita, J. Chem. Phys. 118, 1341 (2003); K. Nishikawa and T. Morita, Chem. Phys. Lett. 316, 238 (2000).

${ }^{17}$ K. Nishikawa, H. Ochiai, K. Saitow, and T. Morita, Chem. Phys. 286, 421 (2003).

${ }^{18}$ K. Saitow, D. Kajiya, and K. Nishikawa, J. Am. Chem. Soc. 126, 422 (2004).

${ }^{19}$ M. Kamiya, K. Muroki, and M. Uematsu, J. Chem. Thermodyn. 27, 337 (1995).

${ }^{20}$ C. A. Eckert, D. H. Ziger, K. P. Johnston, and S. J. Kim, J. Phys. Chem. 90, 2738 (1986).

${ }^{21}$ P. G. Debenedetti, Chem. Eng. Sci. 42, 2203 (1987).

${ }^{22}$ E. F. Carome, C. B. Cykowski, J. F. Havlice, and D. A. Swyt, Physica (Amsterdam) 38, 307 (1968).

${ }^{23}$ Z. Chen, K. Tozaki, and K. Nishikawa, Jpn. J. Appl. Phys., Part 1 38, 6840 (1999).

${ }^{24}$ D. Kajiya, K. Nishikawa, and K. Saitow, J. Phys. Chem. A 109, 7365 (2005)

${ }^{25}$ P. G. Debenedetti and R. S. Mohamed, J. Chem. Phys. 90, 4528 (1989).

${ }^{26}$ I. B. Petsche and P. G. Debenedetti, J. Phys. Chem. 95, 386 (1991).

${ }^{27}$ R. G. Alargova, S. Deguchi, and K. Tsujii, Colloids Surf., A 183-185, 303 (2001).

${ }^{28}$ R. G. Alargova and K. Tsujii, Prog. Colloid Polym. Sci. 126, 134 (2004).

${ }^{29}$ S. K. Ghosh, R. G. Alargova, S. Deguchi, and K. Tsujii, J. Phys. Chem. B 110, 2590 (2006)

${ }^{30}$ S. Mukai, S. Deguchi, and K. Tsujii, Colloids Surf., A 282-283, 483 (2006)

${ }^{31}$ S. Deguchi and K. Tsujii, Soft Matter 3, 797 (2007).

${ }^{32}$ S. K. Ghosh and K. Tsujii, J. Phys. Chem. B 112, 6906 (2008).

${ }^{33}$ S. K. Ghosh, J. Phys. Chem. B 113, 3253 (2009).

${ }^{34}$ S. K. Ghosh, S. Deguchi, S. Mukai, and K. Tsujii, J. Phys. Chem. B 111, 8169 (2007)
${ }^{35}$ Dynamic Light Scattering, edited by R. Pecora (Plenum, New York, 1985).

${ }^{36}$ Y. Hiejima and M. Yao, J. Chem. Phys. 119, 7931 (2003).

${ }^{37}$ H. E. Dillon and S. G. Penoncello, Int. J. Thermophys. 25, 321 (2004).

${ }^{38}$ See supplementary material at http://dx.doi.org/10.1063/1.3373404 for the temperature and the pressure dependence of the refractive index of ethanol calculated by Eq. (5).

${ }^{39}$ K. S. Schmitz, An Introduction to Dynamic Light Scattering by Macromolecules (Academic, New York, 1990).

${ }^{40}$ S. B. Kiselev, J. F. Ely, I. M. Abdulagatov, and M. L. Huber, Ind. Eng. Chem. Res. 44, 6916 (2005).

${ }^{41}$ T. Clifford, Fundamentals of Supercritical Fluids (Oxford University Press, New York, 1999).

${ }^{42}$ A. R. Bazaev, I. M. Abdulagatov, E. A. Bazaev, and A. Abdurashidova, Int. J. Thermophys. 28, 194 (2007).

${ }^{43}$ I. F. Golubev, Viscosity of Gases and Gas Mixtures. A Handbook (Israel Program for Scientific Translations, Jerusalem, 1970).

${ }^{44}$ H. Y. Lo and L. I. Stiel, Ind. Eng. Chem. Fundam. 8, 713 (1969).

${ }^{45}$ T. J. Butenhoff, M. G. E. Goemans, and S. J. Buelow, J. Phys. Chem. 100, 5982 (1996)

${ }^{46}$ B. V. Derijaguin, Theory of Stability of Colloids and Thin Films (Plenum, New York, 1989).

${ }^{47}$ E. J. Verwey and J. T. Overbeek, Theory of Stability of Lyophobic Colloids (Elsevier, New York, 1948)

${ }^{48}$ R. J. Hunter, Foundations of Colloid Science (Clarendon, Oxford, 1995), Vol. 1.

${ }^{49}$ D. J. Shaw, Introduction to Colloid and Surface Chemistry, 4th ed. (Butterworth-Heinemann, Oxford, 1992).

${ }^{50}$ P. C. Heimenz and R. Rajagopalan, Principles of Colloid and Surface Chemistry, 3rd ed. (Dekker, New York, 1997).

${ }^{51}$ P. F. Evans and H. Wennerström, The Colloidal Domain, 2nd ed. (Wiley, New York, 1999).

${ }^{52} \mathrm{~J}$. Israelachvili, Intermolecular and Surface Forces, 2nd ed. (Academic, New York, 1991).

${ }^{53}$ J. Lyklema, Adv. Colloid Interface Sci. 2, 67 (1968).

${ }^{54}$ A. F. Rogers, Am. Mineral. 13, 73 (1928). 